\title{
Development of Laser Ionization Imaging Mass Spectrometry for Multiple Drugs Administered to Cancer Cells
}

\author{
Hisanao Hazama ${ }^{1}$, Hiroki Kannen ${ }^{1}$, Jun Aoki ${ }^{2}$, Michisato Toyoda ${ }^{2}$, Tatsuya Fujino ${ }^{3}$, Yasufumi Kaneda ${ }^{4}$ \\ and Kunio Awazu ${ }^{1,5,6}$ \\ 1. Graduate School of Engineering, Osaka University, Suita, Osaka, Japan \\ 2. Graduate School of Science, Osaka University, Toyonaka, Osaka, Japan \\ 3. Graduate School of Science and Engineering, Tokyo Metropolitan University, Hachioji, Tokyo, Japan \\ 4. Graduate School of Medicine, Osaka University, Suita, Osaka, Japan \\ 5. Graduate School of Frontier Biosciences, Osaka University, Suita, Osaka, Japan \\ 6. The Center for Advanced Medical Engineering and Informatics, Osaka University, Suita, Osaka, Japan
}

Photodynamic therapy (PDT) is a less invasive treatment of cancer using a photosensitizing drug selectively accumulated in a tumor. By exciting the photosensitizing drug with a laser or other light sources, reactive oxygen species are produced and cancer cells are killed. PDT is expected for the treatment of cancer cells which gained resistance to anticancer drugs, while PDT is not suitable for treatment of a large tumor because the light cannot reach the deep region in the tumor. Thus, the combined therapy using chemotherapy and PDT has been proposed for treatment of a tumor including cancer cells which gained resistance to anticancer drugs. To evaluate and to optimize the efficacy of the combined therapy, it is important to measure and to control the spatial distributions of both the anticancer drug and the photosensitizing drug in the tumor tissue. In imaging mass spectrometry (IMS) using matrix-assisted laser desorption/ionization (MALDI), multiple drugs can be detected simultaneously. However, the detection sensitivity of IMS for drugs is lower than other drug imaging methods such as autoradiography, and some drugs could not effectively be ionized with MALDI. Therefore, a new ionization reagent, zeolite matrix, which is the mixture of zeolite and conventional MALDI matrix, has been developed to enhance the ionization efficiency of MALDI [1-3]. The detection sensitivity for an anticancer drug, docetaxel, was increased by 13-fold with the zeolite matrix [3]. In this research, simultaneous imaging of an anticancer drug and a photosensitizing drug administered to cancer cells was investigated using the zeolite matrix to confirm that the photosensitizing drug could accumulate in anticancer drug resistant cancer cells.

An anticancer drug, docetaxel, and a photosensitizing drug, protoporphyrin IX (PpIX), were dissolved in a cell culture medium at the concentrations of 30 and $5 \mu \mathrm{mol} / \mathrm{L}$, respectively. The human prostate cancer cell line PC-3 and its docetaxel resistant cell line PC-3-DR were cultured in the medium containing the drugs. After 1 hour, cells were washed with phosphate-buffered saline and centrifuged. After that, cell suspensions were prepared by removal of supernatant and addition of distilled water. Each cell suspension containing about 5000 cells with a volume of $1 \mu \mathrm{L}$ was dropped onto a glass slide with a transparent and conductive coating of indium tin oxide (8237001, Bruker Daltonik GmbH, Germany) and dried. Zeolite matrix was made by mixing a zeolite, NaY5.6, with a MALDI matrix, 6-aza-2thiothymine, at 1:1 weight ratio and was dissolved with $50 \%$ acetone in water at a concentration of 10 $\mathrm{mg} / \mathrm{mL} .1 \mu \mathrm{L}$ of the zeolite matrix solution was dropped onto the dried droplet of the cell suspension and dried. Mass spectra were measured using a MALDI time-of-flight mass spectrometer in the positive reflector mode (Voyager-DE PRO, Applied Biosystems, USA) and an external laser source at a wavelength of $355 \mathrm{~nm}$, pulse width of $5 \mathrm{~ns}$, and a pulse repetition rate of $20 \mathrm{~Hz}$. Ion images of the dried droplets were obtained with the scanning mode IMS by automatically controlling the mass spectrometer 
using a software (MMSIT, Novartis, Switzerland).

Figure 1 shows ion images of the sodium adduct ion $[\mathrm{M}+\mathrm{Na}]^{+}$of docetaxel at $\mathrm{m} / \mathrm{z} 829.9$ and protonated ion $[\mathrm{M}+\mathrm{H}]^{+}$of PpIX at $\mathrm{m} / \mathrm{z} 563.3$ simultaneously obtained from the PC-3 and PC-3-DR cells. Ion signal intensity of $[\mathrm{M}+\mathrm{Na}]^{+}$of docetaxel from PC-3 cells was significantly higher than that from PC-3DR cells. It is supposed that docetaxel is ejected from the PC-3-DR cells at a higher rate compared with that of PC-3 and cannot accumulate in the PC-3-DR cells, because the PC-3-DR cells have higher resistance to docetaxel compared with the PC-3 cells. On the other hand, $[\mathrm{M}+\mathrm{H}]^{+}$of PpIX was detected from both PC-3 and PC-3-DR cells with similar ion signal intensities. These results show that PpIX was accumulated in both PC-3 and PC-3-DR cells and that PDT would be effective for treatment of anticancer drug resistant cancer cells. The ion images in this research were measured in the scanning mode IMS at a spatial resolution of about $100 \mu \mathrm{m}$. However, cellular scale observation is preferable to distinguish the cancer cells with and without resistance to the anticancer drug in tumor tissues. Therefore, as the next step, cellular scale observation will be performed using a stigmatic mode imaging mass spectrometer with a spatial resolution of $1 \mu \mathrm{m}[4-6]$.

References:

[1] Y Komori et al., J. Phys. Chem. 114 (2010) p. 1593.

[2] R Yamamoto and T Fujino, Chem. Phys. Lett. 543 (2012) p. 76.

[3] H Kannen et al., Int. J. Mol. Sci. 15 (2014) p. 11234.

[4] H Hazama et al., J. Biomed. Opt. 16 (2011) 046007, p. 1.

[5] J Aoki and M Toyoda, J. Mass Spectrom. Soc. Jpn. 61 (2013) p. 23.

[6] J Aoki et al., J. Phys. Soc. Jpn. 83 (2014) 023001, p. 1.

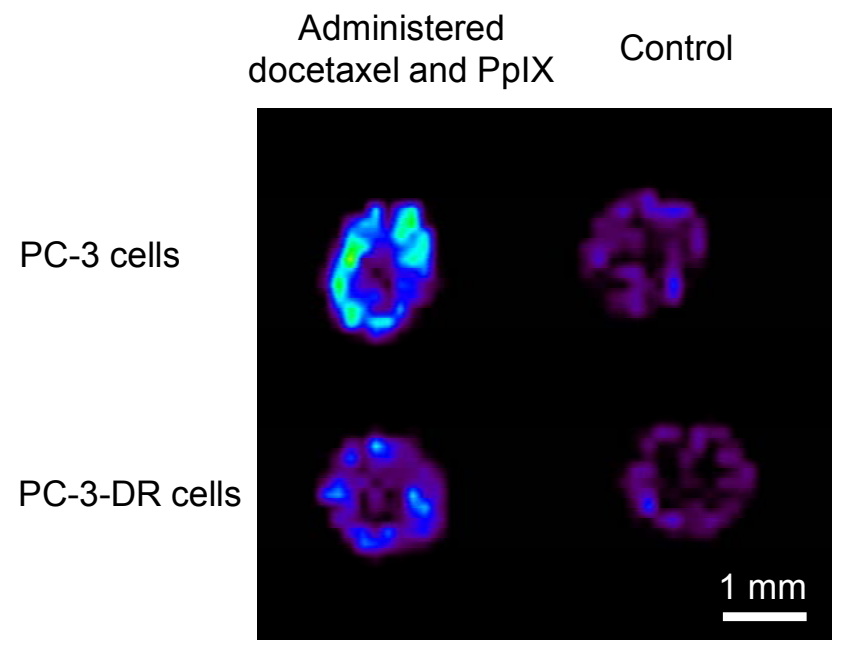

(a) docetaxel

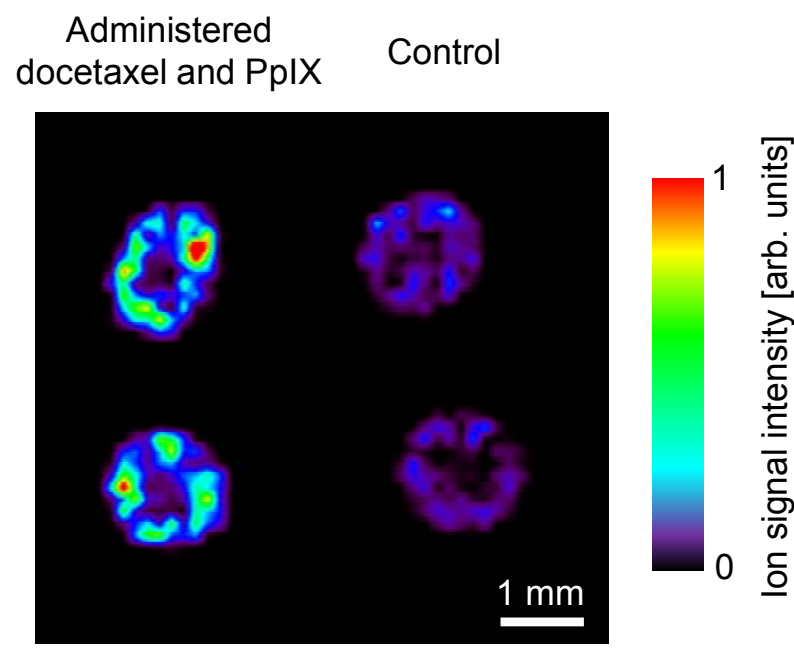

(b) PplX

Figure 1. Ion images of (a) sodium adduct ion $[\mathrm{M}+\mathrm{Na}]^{+}$of docetaxel at $\mathrm{m} / \mathrm{z} 829.9$ and (b) protonated ion $[\mathrm{M}+\mathrm{H}]^{+}$of PpIX at $\mathrm{m} / \mathrm{z} 563.3$ obtained from the human prostate cancer cell line PC-3 and its docetaxel resistant cell line PC-3-DR. Control means the cells without administration of the drugs. 